\title{
Analyse gratuite de votre portefeuille de titres
}

Pour quelle raison? En principe vous êtes satisfait de la gestion de votre banque.

\section{Vous êtes-vous déjà posé la question, que pouvez-vous attendre d'un dépôt de titres?}

- Pendant les bonnes années boursières votre dépôt de titres a pris de la valeur, par contre lors d'un crash boursier vous devez accepter des pertes. Est-ce que votre gérant de fortune est bien indemnisé par ses frais de gestion mais a également bien géré votre portefeuille? A-t-il pu lors d'une année boursière record atteindre des performances au-dessus de la moyenne du marché et dans les mauvaises années a-t-il su limiter les pertes?

- Votre dépôt de titres est-il composé dans sa majorité de fonds de placements de votre propre banque dont les frais sont élevés et/ou pas transparents?

- Est-ce que votre dépôt de titres correspond à vos besoins et vos attentes ou est-il investi dans sa majorité, par exemple, dans des monnaies étrangères, valeurs que vous ne souhaitez en principe pas?

- Est-ce que votre dépôt est bien diversifié ou est-il investi dans peu de titres et cela représente un risque élevé inutile qui pourrait être évité?

Les frais imputés sur votre portefeuille de titres peuvent réduire votre performance d'une manière importante

L'exemple suivant vous montre que coûte $1 \%$ par année de frais trop facturés pendant 20 ans

\begin{tabular}{|c|c|c|c|}
\hline Investition & Durée & $\varnothing$ Intérêts & Capital final \\
\hline Fr. 250000.- & 20 ans & $7 \%$ & Fr. 967421.- \\
\hline Fr. 250000.- & 20 ans & $6 \%$ & Fr. 801 784.- \\
\hline \multicolumn{2}{r|}{ Ce montant a été prélevé par la banque sur votre bénéfice: } & Fr. 165637.- \\
\cline { 2 - 4 }
\end{tabular}

Prenez garde à ce que votre banque ne vous prélève pas trop de frais de tous genres qui diminuent votre performance boursière. Profitez de notre analyse gratuite de votre portefeuille de titres et envoyez-nous le talon réponse encore aujourd'hui.

\section{Talon réponse}

Prière de l'envoyer ou par fax au: 0319595010

Prénom / Nom

Adresse

NPA / Lieu

Date de naissance

Téléphone privé / cabinet

Atteignable le plus facilement (heure)

Adresse e-mail

Je désire une analyse gratuite et sans engagement de mon dépôt de titres. Prière de $m$ 'appeler pour discuter du déroulement de l'analyse.

Je m'intéresse aux thèmes suivants:
O $3^{\mathrm{e}}$ pilier a
O Responsabilité civile prof.
O Prévoyance LPP
O Placement avec garantie
$O$ Hypothèque
O Protection juridique

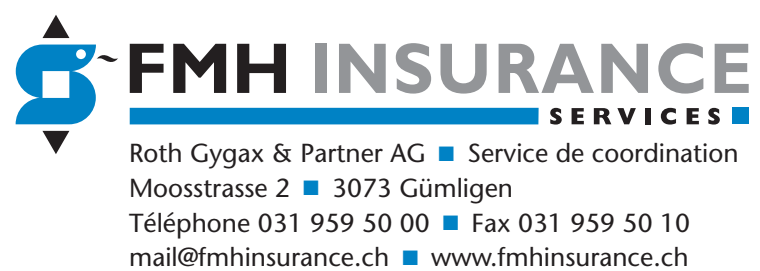

